\title{
Video Surveillance for Effective Object Detection with Alarm Triggering
}

\author{
${ }^{1}$ B. Priyanka,${ }^{2}$ A. Ramya,${ }^{3}$ J. Sri Katheswari \\ ${ }^{1,2,3}$ Dept.of Information Technology Annai Teresa College Of Engineering,Thirunavalur-607204, \\ Ulundurpet TK, Villupuram DT.
}

\begin{abstract}
This paper presents a novel algorithm for detection and segmentation of foreground objects from a video which contains both stationary and moving background objects and under-goes both gradual and sudden "once-off" changes with fixed background and static cameras. Our method achieves complete detection of moving objects by involving three significant proposed modules: a background modeling (BM) module, an alarm trigger (AT) module, and an object extraction $(O E)$ module For our proposed BM module, a unique twophase background matching procedure is performed using rapid matching followed by accurate matching in order to produce optimum background pixels for the background model. Next, our proposed AT module eliminates the unnecessary examination of the entire background region, allowing the subsequent OE module to only process blocks containing moving objects. And the alarm triggers while a new object enters into the frame, the foreground information and background information are identified using the reference frame as background model. Here, both visual as well as quantitative measures show an improved performance and the scheme has a strong potential for applications in real time surveillance.
\end{abstract}

Index Terms: Background modeling, background subtraction, video segmentation, video surveillance, Alarm Triggering , Shadow Removal.

\section{Introduction}

Object detection and tracking in video is a challenging problem and has been extensively investigated in the past two decades.(i) Video Surveillance: recognition system process image sequences captured by video cameras monitoring sensitive areas such as bank, departmental stores, parking lots and country border to determine whether one or more humans engaged are suspicious or under criminal activity. (ii) Content based video retrieval: A human behavior understanding system scan an input video, and an action or event specified in high-level language as output. The former involves locating object in the frames of a video sequence, while the latter represents the process of monitoring the object's spatial and temporal changes in each frame.

A popular approach called background subtraction is used in this scenario, where moving objects in a scene can be obtained by comparing each frame of the video with a background [1]. Presently, an additional step is carried out to remove these misclassified objects and shadows for effective object detection. To alleviate this problem, we propose a simple but efficient object detection technique, which is invariant to change in illumination and motion in the background. The proposed approach also neutralizes the presence of shadows in detected objects. In all these applications fixed cameras are used with respect to static background (e.g. stationary surveillance camera) and a common approach of background subtraction is used to obtain an initial estimate of moving objects.

\section{Related Work}

For object detection in surveillance system, background modeling plays a vital role. Wren et al. have proposed to model the background independently at each pixel location which is based on computation of Gaussian probability density function (pdf) on the previous pixel values [2] Stauffer and Grimson developed a complex procedure to accommodate permanent changes in the background scene [3]. Here each pixel is modeled separately by a mixture of three to five Gaussians. TheW4model presented by Haritaoglu et al. is a simple and effective method [4]. It uses three values to represent each pixel in the background image namely, the minimum intensity, the maximum intensity, and the maximum intensity difference between consecutive frames of the training sequence. From the existing literature, it is observed that most of the simple schemes are ineffective on videos with illumination variations, motion in background, and dynamically textured indoor and outdoor environment etc. Recently, several other methods suitable for a variety of background situations have been proposed. Among them, Mixture of Gaussians (MoG) [11,9] is considered as a promising method. In MoG, the colors from a pixel in a background object are described by multiple Gaussian distributions. Good foreground object detection results were reported by applying MoG to outdoor scenes. Further investigations $b$ showed that MoG with more than two Gaussians can degrade the performance in foreground object detection. The background variation model employed in $W 4$ [3] is a generalization of the Gaussian model. In [12], Toyama 
et al employed a linear Wiener filter to learn and predict color changes in each background pixel through the video.

\section{The Proposed Libs Scheme}

The LIBS scheme consists of two stages. The first stage deals with finding the stationary pixels in the frames required for background modeling, followed by defining the intensity range from those pixels. In the second stage a local threshold based background subtraction method tries to find the objects by comparing the frames with the established background. LIBS uses two parameters namely, window size (an odd length window) and a constant for its computation. The optimal values are selected experimentally. Both stages of LIBS scheme are described as follows.

\section{A) BAYES CLASSIFICATION OF BACKGROUND AND FOREGROUND \\ 2.1 Problem Specification}

For the general purpose of video processing, the background is usually considered as the scene without the presence of objects of interest, such as human objects or moving vehicles. Background is usually composed of non-living objects that remain passively in the scene. In a video about a general environment, the background can consist of both stationary and moving objects. Meanwhile, the background might be undergoing two types of changes over the time. One is the gradual changes caused by natural lighting variations, e.g., the change of illumination from day to night. In this paper, we propose a general Bayesian framework which can integrate multiple features to model the background for foreground object detection.

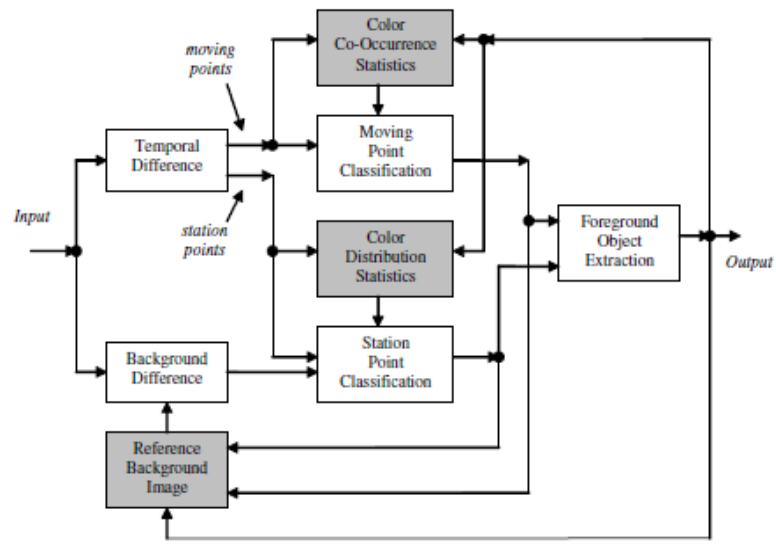

Figure 1: The block diagram of the algorithm.

\section{Algorithm Description}

It consists of four parts: In the first step, non-change pixels in the image stream are filtered out by using simple background and temporal differences. The detected changes are separated as pixels belonging to stationary and moving objects according to inter-frame changes. In the second step, the pixels associated with stationary or moving objects are further classified as background or foreground based on the learned statistics of colors and color co-occurrences respectively by using the Bayes decision rule. In the third step, foreground objects are segmented by combining the classification results from both stationary and moving parts. In the fourth step, background models are updated.

\section{A. Development of Background Model}

Conventionally, the first frame or a combination of first few frames is considered as the background model. However, this model is susceptible to illumination variation, dynamic objects in the background, and also to small changes in the background like waving of leaves etc. A number of solutions to such problems are reported, where the background model is frequently updated at higher computational cost and thereby making them unsuitable for real time deployment.

Here the RGB frame sequences of a video are converted to gray level frames. Initially, few frames are considered for background modeling and pixels in these frames are classified as stationary or non-stationary by analyzing their deviations from the mean. The background is then modeled taking all the stationary pixels into account. Background model thus developed, defines a range of values for each background pixel location The steps of the proposed background modeling are presented in Algorithm 1. 


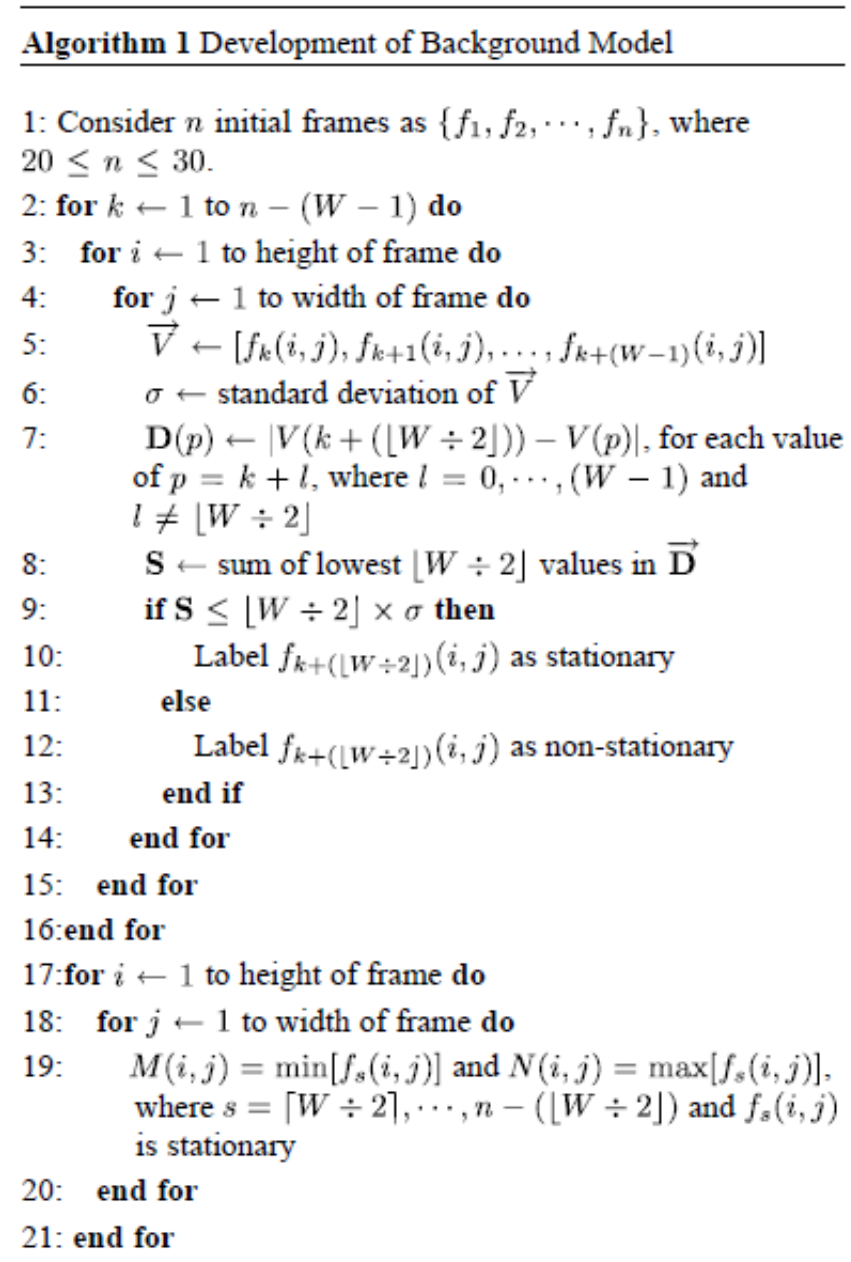

\section{B. Extraction of Foreground Object}

After successfully developing the background model, a local thresholding based background subtraction is used to find the foreground objects. A constant is considered that helps in computing the local lower threshold and the local upper threshold. These local thresholds help in successful detection of objects suppressing shadows if any. The steps of the algorithm are outlined in Algorithm2.

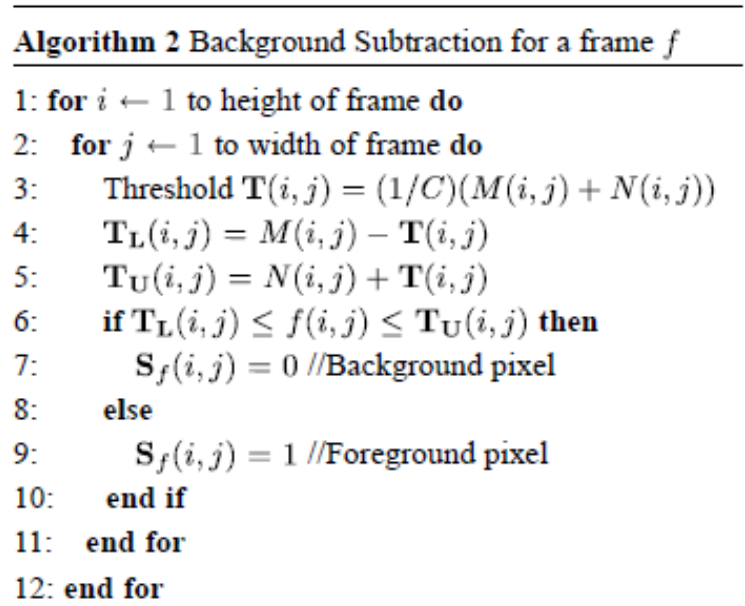

\section{Alarm Trigger Module}

This module consists of a novel block-based entropy evaluation method developed for the employment of block candidates, after which the most likely moving objects within the motion blocks are determined based on block based morphological erosion and dilation operations. 


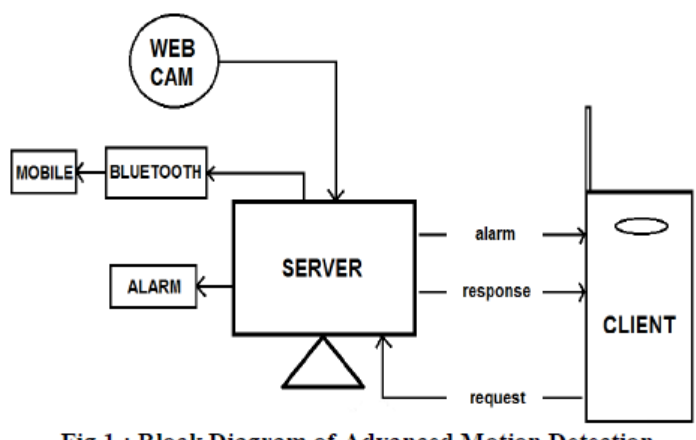

Fig 1 : Block Diagram of Advanced Motion Detection Algorithm for Patient Monitoring Using Cell Phone for Videc Display

\section{Image Description}

To show the efficacy of the proposed LIBS scheme, simulation has been carried out on different recorded video sequences namely, "Time of Day", "PETS2001", "Intelligent Room", "Campus", "Fountain", and "Lobby".

For comparative analysis, the above video sequences are simulated with the proposed LIBS scheme and three other existing schemes namely, Gaussian mixture model (GMM) [13], expected Gaussian mixture model (EGMM) [14], and model of Reddy et al. [9]. Percentage of correct classification(PCC)

$$
P C C=\frac{T P+T N}{T P F} \times 100
$$

where TP is true positive that represents the number of correctly detected foreground pixels and TN is true negative representing the number of correctly detected background pixels.TPF represents the total number of pixels in the frame. TP and TF are measured from a predefined ground truth.
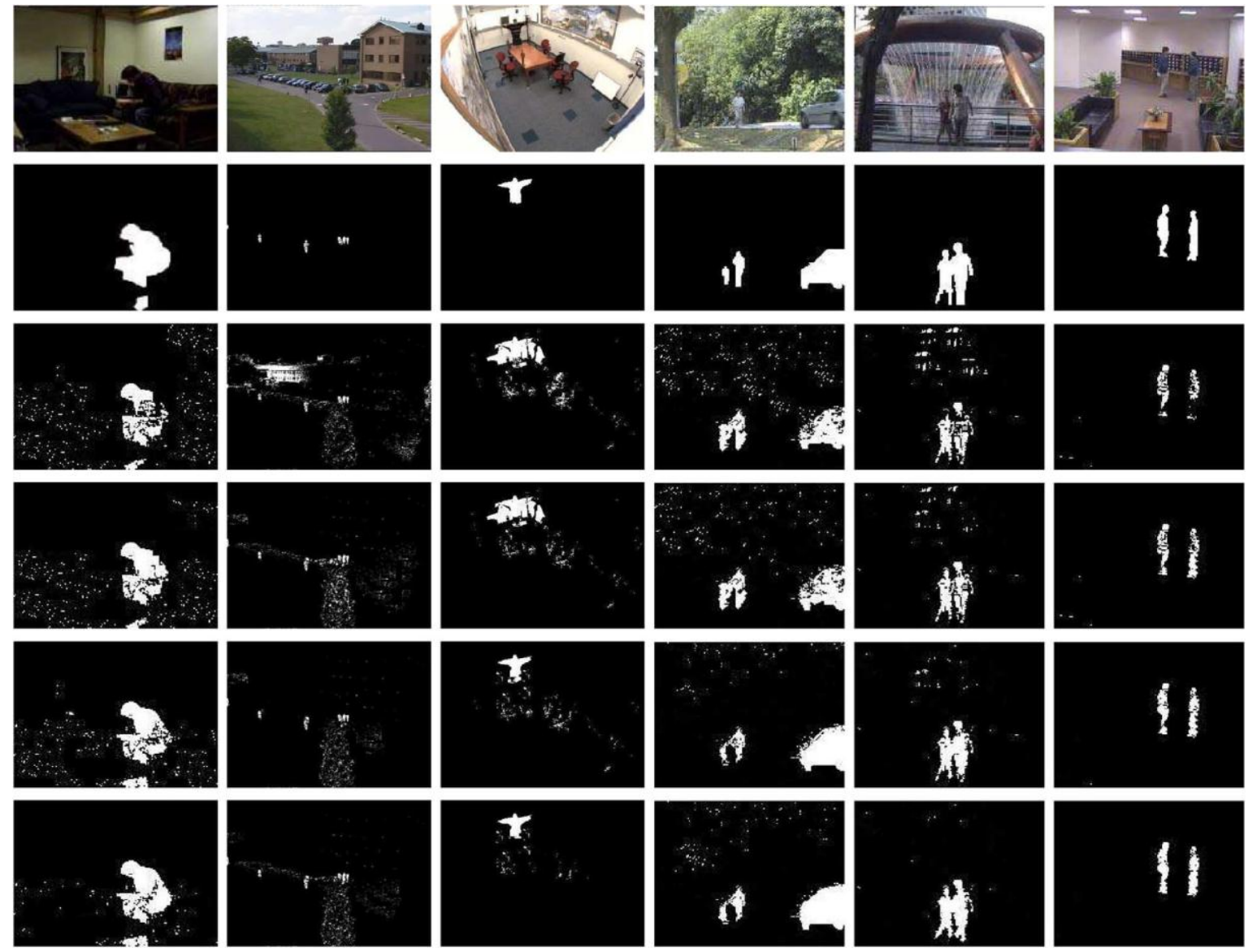


\section{Conclusion}

In this work we have proposed a simple but robust scheme of background modeling and local threshold based object detection . It is then applied to both stationary and moving background objects with suitable feature vectors. Different feature vectors are selected for different background parts. Foreground objects are segmented by fusing the results from both station and motion pixels. A Bayes decision rule for background and foreground classification from a general feature vector is established. It is then applied to both stationary and moving background objects with suitable feature vectors. Different feature vectors are selected for different background parts. Foreground objects are segmented by fusing the results from both station and motion pixels.

\section{References}

[1] A. Yilmaz, O. Javed, and M. Shah, "Object tracking: A survey,” ACM Comput. Surv., vol. 38, Dec. 2006.

[2] C.Wren, A. Azarbayejani, T. Darrell, and A. Pentland, "Pfinder: Realtime tracking of the human body," IEEE Trans. Patt. Anal. Mach. Intell., vol. 19, no. 7, pp. 780-785, Jul. 1997.

[3] C. Stauffer and W. Grimson, “Adaptive background mixture models for real-time tracking,” in IEEE Comput. Soc. Conf. CVPR, 1999, pp. 246-252.

[4] I. Haritaoglu, D. Harwood, and L. Davis, "W4: Real-time surveillance of people and their activities," IEEE Trans. Patt. Anal. Mach. Intell., vol. 22, no. 8, pp. 809-830, Aug. 2000.

[5] J. Jacques, C. Jung, and S. Musse, "Background subtraction and shadow detection in grayscale video sequences," in Eighteenth Brazilian Symp. Computer Graphics and Image Processing, Oct.2005, pp. 189-196.

[6] J.McHugh, J. Konrad,V. Saligrama, and P. Jodoin, "Foreground-adaptive background subtraction," IEEE Signal Process.Letters, vol. 16, no. 5, pp. 390-393, May 2009.

[7] O. Barnich and M. Van Droogenbroeck, "ViBe: A universal background subtraction algorithm for video sequences," IEEE Trans. Image Process., vol. 20, no. 6, pp. 1709-1724, Jun. 2011.

[8] W. Kim and C. Kim, "Background subtraction for dynamic texture scenes using fuzzy color histograms," IEEE Signal Process. Lett., vol. 19, no. 3, pp. 127-130, Mar. 2012.

[9] V. Reddy, C. Sanderson, and B. Lovell, "Improved foreground detection via block-based classifier cascade with probabilistic decision integration,’IEEE Trans. Circuits Syst. Video Technol., vol. 23, no. 1, pp. 83-93, Jan. 2013.

[10] E. Salvador, A. Cavallaro, and T. Ebrahimi, “Cast shadow segmentation using invariant color features," Comput. Vis. Image Understand., vol. 95, no. 2, pp. 238-259, 2004.

[11] J. Choi, Y. Yoo, and J. Choi, "Adaptive shadow estimator for removing shadow of moving object," Comput. Vis. Image Understand., vol. 114, no. 9, pp. 1017-1029, 2010.

[12] Z. Liu, K. Huang, and T. Tan, "Cast shadow removal in a hierarchical manner using MRF," IEEE Trans. Circuits Syst. Video Technol., vol. 22, no. 1, pp. 56-66, Jan. 2012.

[13] L. Li,W.Huang, I.Y.H.Gu, andQ. Tian, "Foreground object detection from videos containing complex background," in Proc. Eleventh ACM Int. Conf. Multimedia, Nov. 2003, pp. 2-10.

[14] Z. Zivkovic, "Improved adaptive Gausian mixture model for background subtraction," in Proc. IEEE Int. Conf Pattern Recognition, Aug. 2004, pp. 28-31. 